\title{
SOLVABILITY OF THE NONLINEAR BOUNDARY VALUE PROBLEMS USING THE GREEN FUNCTION
}

\author{
Ngo Thi Kim Quy \\ $T N U$ - University of Economics and Business Administration
}

\begin{abstract}
The Green function has wide applications in the study of boundary value problems. In particular, the Green function is an important tool to show the existence and uniqueness of problems. In this paper, we study solvability of nonlinear boundary problems using the Green function. Differently from other authors, we reduce the problem to an operator equation for the right-hand side function. Consider this function in a specified bounded domain, we prove the contraction of the operator. This guarantees the existence and uniqueness of a solution of the problem.
\end{abstract}

Key words: Green function; boundary value problem; nonlinear; existence; uniqueness of solution.

\section{TÍNH GIẢI ĐƯợC CỦA BÀI TOÁN GIÁ TRI BIÊN PHI TUYẾN SỬ DỤNG HÀM GREEN}

Ngô Thị Kim Quy

Truòng Đại học Kinh tế và Quản trị Kinh doanh - ĐH Thái Nguyên

\section{TÓM TẮT}

Hàm Green có ứng dụng rộng rãi trong nghiên cứu các bài toán giá trị biên. Đặc biệt, hàm Green là công cụ quan trọng để chỉ ra sự tồn tại và duy nhất nghiệm của các bài toán. Trong bài báo này, chúng tôi nghiên cứu tính giải được của bài toán biên phi tuyến đối với phương trình vi phân có sử dụng hàm Green. Khác với cách tiếp cận của các tác giả khác, chúng tôi đưa bài toán ban đầu về phương trình toán tử đối với hàm vế phải. Xét hàm này trong miền bị chặn xác định, với một số điều kiện dễ kiểm tra chứng tỏ rằng toán tử này có tính chất co. Điều này bảo đảm bài toán gốc có nghiệm duy nhất.

Từ khóa: Hàm Green; bài toán giá trị biên; phi tuyến; tồn tại; duy nhất nghiệm.

Ngày nhận bài: 03/02/2020; Ngày hoàn thiện: 27/02/2020; Ngày đăng: 29/02/2020

Email: kimquykttn@gmail.com

https://doi.org/10.34238/tnu-jst.2020.02.2581 


\section{Introduction}

The nonlinear ordinary differential equations with different types of boundary conditions have been studied intensively since the eighties of the last century. The reason for this phenomenon is that these equations describe the deformations of elastic beams under nonlinear loads with different constrains at ends. There have been many research results on the qualitive aspects of the problems such as existence, uniqueness and positivity of solutions, where the upper and lower solution method, the variational method, the methods of fixed point theorems are used. Differently from other authors, we reduce the problem to an operator equation for the right-hand side function. Under some easily verified conditions on this function in a specified bounded domain, we prove the contraction of the operator. This guarantees the existence and uniqueness of a solution of the problem.

\section{Green function for some problems}

Consider the linear homogeneous boundaryvalue problem

$$
\begin{aligned}
& L[y(x)] \\
& \equiv p_{0}(x) \frac{d^{n} y}{d x^{n}}+p_{1}(x) \frac{d^{n-1} y}{d x^{n-1}}+\ldots+p_{n}(x) y=0, \\
& M_{i}(y(a), y(b)) \equiv \\
& \sum_{k=0}^{n-1}\left(\alpha_{k}^{i} \frac{d^{k} y(a)}{d x^{k}}+\beta_{k}^{i} \frac{d^{k} y(b)}{d x^{k}}\right)=0, \\
& (i=1, \ldots, n)
\end{aligned}
$$

which is assumed well-posed on the interval $(a, b)$. The coefficients of the equation $p_{i}(x), i=0, \ldots, n$, are continuous functions on $(a, b)$, where the leading coefficient $p_{0}(x)$ must be non-zero in all points in $(a, b)$.
Definition 1. (see [1] ) The function $G(x, t)$ is said to be the Green function for the boundary value problem in (1) and (2), if, as a function of its first variable $x$, it meets the following defining criteria, for any $t \in(a, b)$.

(i) On both intervals $[a, t)$ and $(t, b], G(x, t)$ is a continuous function having continuous derivatives up to $\mathrm{n}^{\text {th }}$ order, and satisfies the governing equation in (1) on $(a, t)$ and $(t, b)$, i.e.:

$L[G(x, t)]=0, x \in(a, t) ;$

$L[G(x, t)]=0, x \in(t, b)$.

(ii) $G(x, t)$ satisfies the boundary conditions in (2), i.e.:

$$
M_{i}(G(a, t), G(t, b))=0, i=1, \ldots, n .
$$

(iii) For $x=t, G(x, t)$ and all its derivatives up to $n-2$ are continuous

$$
\begin{gathered}
\lim _{x \rightarrow t^{+}} \frac{\partial^{k} G(x, t)}{\partial x^{k}}-\lim _{x \rightarrow t^{-}} \frac{\partial^{k} G(x, t)}{\partial x^{k}}=0, \\
k=0, \ldots, n-2 .
\end{gathered}
$$

(iv) The $(n-1)$ derivative of $G(x, t)$ is discontinuous when $x=t$, providing $\lim _{x \rightarrow t^{+}} \frac{\partial^{n-1} G(x, t)}{\partial x^{n-1}}-\lim _{x \rightarrow t^{-}} \frac{\partial^{n-1} G(x, t)}{\partial x^{n-1}}=-\frac{1}{p_{0}(t)}$.

Theorem 1. (see [1]) (existence and uniqueness). If the homogeneous boundaryvalue problem in (1) and (2) has only a trivial solution, then there exists an unique Green function $G(x, t)$ associated with the problem.

Consider the linear inhomogeneous equation $L[y(x)]$

$\equiv p_{0}(x) \frac{d^{n} y}{d x^{n}}+p_{1}(x) \frac{d^{n-1} y}{d x^{n-1}}+\ldots+p_{n}(x) y=-f(x)$,

subject to the homogeneous boundary conditions 


$$
\begin{aligned}
& M_{i}(y(a), y(b)) \equiv \\
& \sum_{k=0}^{n-1}\left(\alpha_{k}^{i} \frac{d^{k} y(a)}{d x^{k}}+\beta_{k}^{i} \frac{d^{k} y(b)}{d x^{k}}\right)=0, \\
& (i=1, \ldots, n)
\end{aligned}
$$

where the coefficients $p_{j}(x)$ and the righthand side term $f(x)$ in the governing equation are continuous functions, with $p_{0}(x) \neq 0 \quad$ on $(a, b)$, and $M_{i}$ represent linearly independent forms with constant coefficients.

Theorem 2. (see [1]) If the boundary-value problem stated by the inhomogeneous equation in (3) subject to the homogeneous conditions in (4) is well-posed, then the unique solution for (3) and (4) can be expressed by the integral

$$
y(x)=\int_{a}^{b} G(x, t) f(t) d t
$$

whose kernel $G(x, t)$ is the Green function of the corresponding homogeneous problem.

Example 1. Considered boundary value problem

$$
\begin{gathered}
u^{(4)}(x)=g(x), \quad 0<x<1, \\
u(0)=u^{\prime}(0)=u^{\prime}(1)=u^{\prime \prime \prime}(1)=0 .
\end{gathered}
$$

The general expression of a Green function for the homogeneous $4^{\text {th }}$ order differential equation.

$$
G(x, t)=\left\{\begin{array}{r}
A_{1}+A_{2} x+A_{3} x^{2}+A_{4} x^{3} \text { if } 0 \leq x \leq t \leq 1 \\
B_{1}+B_{2}(1-x)+B_{3}(1-x)^{2}+B_{4}(1-x)^{3} \\
\text { if } 0 \leq t<x \leq 1
\end{array}\right.
$$

where $A_{1}, A_{2}, A_{3}, A_{4}$ and $B_{1}, B_{2}, B_{3}, B_{4}$ are functions of $t$. Knowing that the Green function $G(x, t)$ satisfies the condition (6) we have

$$
A_{1}=A_{2}=B_{2}=B_{4}=0 .
$$

We deduce that the Green function for the problem

$$
G(x, t)=\left\{\begin{array}{l}
A_{3} x^{2}+A_{4} x^{3} \quad \text { if } 0 \leq x \leq t \leq 1 \\
B_{1}+B_{3}(1-x)^{2} \quad \text { if } 0 \leq t<x \leq 1
\end{array}\right.
$$

The continuity conditions (iii) yield the following equations

$$
\left\{\begin{array}{l}
A_{3} t^{2}+A_{4} t^{3}=B_{1}+B_{3}(1-t)^{2} \\
2 A_{3} t+3 A_{4} t^{2}=-2 B_{3}(1-t) \\
2 A_{3}+6 A_{4} t=2 B_{3}
\end{array}\right.
$$

And the jump condition (iv) gives us the equation

$G_{x x x}\left(t^{+}, t\right)-G_{x x x}\left(t^{-}, t\right)=1$.

Therefor, $-6 A_{4}=1$.

From the above conditions we can solve

$$
\begin{aligned}
& A_{3}=-\frac{t(t-2)}{4}, A_{4}=-\frac{1}{6}, \\
& B_{1}=-\frac{1}{6} t^{3}+\frac{1}{4} t^{2}, B_{3}=-\frac{t^{2}}{4} .
\end{aligned}
$$

So the Green function of the above problem is

$$
G(x, t)=\left\{\begin{array}{l}
-t^{2}\left(2 t-6 x+3 x^{2}\right) / 12 \text { if } 0 \leq t<x \leq 1 \\
-x^{2}\left(3 t^{2}-6 t+2 x\right) / 12 \text { if } 0 \leq x \leq t \leq 1
\end{array}\right.
$$

Example 2. Considered boundary value problem

$$
\begin{aligned}
& u^{(4)}(x)=g(x), \quad 0<x<1, \\
& u(0)=u(1)=u^{\prime \prime}(0)=u^{\prime \prime}(1)=0 .
\end{aligned}
$$

Following the same procedure as in examples (5) and (6) we find the Green function to be

$$
G(x, t)= \begin{cases}\frac{t(x-1)\left(x^{2}-2 x+t^{2}\right)}{6} & \text { if } 0 \leq t<x \leq 1 \\ \frac{x(t-1)\left(t^{2}-2 t+x^{2}\right)}{6} & \text { if } 0 \leq x \leq t \leq 1\end{cases}
$$

Example 3. Considered boundary value problem

$$
\begin{aligned}
& u^{(4)}(x)=g(x), \quad 0<x<1, \\
& u(0)=u^{\prime}(0)=u^{\prime \prime}(0)=u^{\prime \prime}(1)=0 .
\end{aligned}
$$

Following the same procedure as in the example with (5) and (6) we find the Green function to be 
$G(x, t)= \begin{cases}-\frac{t^{3}}{6}+\frac{t^{2} x}{2}-\frac{t x^{2}}{2}+\frac{t x^{3}}{6}, & 0 \leq t \leq x \leq 1 \\ \frac{t x^{3}}{6}-\frac{x^{3}}{6}, & 0 \leq x \leq t \leq 1\end{cases}$

\section{The existence and uniqueness of a solution}

In this section, using the contraction mapping principle for an operator equation for the right-hand side we prove the existence and uniqueness of a solution of the problem. This idea of the reduction of boundary value problem to operator equation for right-hand side function was used by ourselves in a previous paper [2] when studying the fourthorder non-linear boundary value problem with other boundary conditions.

Example 4. Considered the fully fourth order boundary value problem

$$
\begin{aligned}
& u^{(4)}(x)=f\left(x, u(x), u^{\prime}(x), u^{\prime \prime}(x), u^{\prime \prime \prime}(x)\right), \\
& (0<x<1) \\
& u(0)=u^{\prime}(0)=u^{\prime}(1)=u^{\prime \prime \prime}(1)=0 .
\end{aligned}
$$

where $f:[0,1] \times i^{4} \rightarrow i$ is continuous.

To investigate the problem (11), (12), for $u \in C^{4}[0,1]$ we set

$$
\varphi(x)=f(x, u(x), y(x), v(x), z(x)) .
$$

Then problem becomes

$$
\begin{aligned}
& u^{(4)}(x)=\varphi(x), \quad 0<x<1, \\
& u(0)=u^{\prime}(0)=u^{\prime}(1)=u^{\prime \prime \prime}(1)=0 .
\end{aligned}
$$

It has a unique solution

$$
u(x)=\int_{0}^{1} G(x, t) \varphi(t) d t
$$

where $G(x, t)$ is the Green function

$$
G(x, t)=\left\{\begin{array}{l}
-t^{2}\left(2 t-6 x+3 x^{2}\right) / 12 \text { if } 0 \leq t<x \leq 1 \\
-x^{2}\left(3 t^{2}-6 t+2 x\right) / 12 \text { if } 0 \leq x \leq t \leq 1
\end{array}\right.
$$

From (14) it follows

$$
u^{\prime}(x)=\int_{0}^{1} G_{x}(x, t) \varphi(t) d t
$$

$$
\begin{aligned}
u^{\prime \prime}(x) & =\int_{0}^{1} G_{x x}(x, t) \varphi(t) d t \\
u^{\prime \prime \prime}(x) & =\int_{0}^{1} G_{x x x}(x, t) \varphi(t) d t
\end{aligned}
$$

where

$G_{x}(x, t)= \begin{cases}\frac{1}{2} t^{2}(1-x) & \text { if } 0 \leq t<x \leq 1 \\ -\frac{1}{2} x\left(t^{2}-2 t+x\right) & \text { if } 0 \leq x \leq t \leq 1\end{cases}$

$G_{x x}(x, t)=\left\{\begin{array}{lr}-\frac{1}{2} t^{2} & \text { if } 0 \leq t<x \leq 1 \\ -\frac{1}{2} t^{2}+t-x & \text { if } 0 \leq x \leq t \leq 1\end{array}\right.$

$G_{x x x}(x, t)= \begin{cases}0 & \text { if } 0 \leq t<x \leq 1 \\ -1 & \text { if } 0 \leq x \leq t \leq 1\end{cases}$

It is easy verify that

$\int_{0}^{1}|G(x, t)| d s \leq \frac{1}{24}, \int_{0}^{1}\left|G_{x}(x, t)\right| d s \leq 0,3577$

$\int_{0}^{1}\left|G_{x x}(x, t)\right| d s \leq \frac{2}{3}, \int_{0}^{1}\left|G_{x x x}(x, t)\right| d s \leq 1$.

Clearly, the solutions $u$ of the problems (14)-(15) depend on $\varphi$, that is, $u=u_{\varphi}(x)$. Therefore, for $\varphi$ we have the equation $\varphi=A \varphi$,

where $A$ is a nonlinear operator defined by

$$
A \varphi(x)=f\left(x, u_{\varphi}(x), y_{\varphi}(x), v_{\varphi}(x), z_{\varphi}(x)\right) \text {, }
$$

with

$$
y_{\varphi}(x)=u_{\varphi}^{\prime}(x), v_{\varphi}(x)=u^{\prime \prime}(x), z_{\varphi}(x)=u^{\prime \prime{ }_{\varphi}}(x) \text {. }
$$

Now, for each number $M>0$ denote

$$
\begin{array}{r}
D_{M}=\left\{(x, u, y, v, z)|0<x<1,| u \mid \leq \frac{M}{24},\right. \\
\left.|y| \leq 0,3577 M,|v| \leq \frac{2 M}{3},|z| \leq M\right\}
\end{array}
$$

and by $B[O, M]$ we denote a closed ball centered at $\mathrm{O}$ with the radius $M$ in the space of continuous functions $C[0,1]$ with the norm 


$$
\|\varphi\|=\max _{0 \leq x \leq 1}|\varphi(x)| .
$$

\section{Theorem 3.}

Suppose that there exists a number $M>0$ such that the function $f(x, u, y, v, z)$ is continuous and

$$
|f(x, u, y, v, z)| \leq M,
$$

Then, the problem (11)-(12) has a solution satisfying the estimates

$$
|u| \leq \frac{M}{24},\left|u^{\prime}\right| \leq 0,3577 M,\left|u^{\prime \prime}\right| \leq \frac{2 M}{3},\left|u^{\prime \prime \prime}\right| \leq M .
$$

Proof. Since the original problem (11)-(12) is reduced to the operator equation (19), the theorem will be proved if we show that this operator equation has a solution. For this purpose, first we show that the operator $A$ defined by (20) maps the closed ball $B[0, M]$ into itself.

Indeed, let $\varphi$ be an element in $\mathrm{B}[\mathrm{O}, \mathrm{M}]$.

Then from (17)-(18) it is easy to obtain

$$
\begin{aligned}
& \|u\| \leq \frac{\|\varphi\|}{24},\|y\| \leq 0,3577\|\varphi\|, \\
& \|v\| \leq \frac{2\|\varphi\|}{3},\|z\| \leq\|\varphi\| .
\end{aligned}
$$

Taking into account (24) and $\|\varphi\| \leq M$ we have

$$
\begin{aligned}
& \|u\| \leq \frac{M}{24},\|y\| \leq 0,3577 M, \\
& \|v\| \leq \frac{2 M}{3},\|z\| \leq M .
\end{aligned}
$$

Therefore, $(x, u, y, v, z) \in D_{M}$ for $x \in[0,1]$. From the definition of $A$ by (19), (20) and the condition (22), we have $A \varphi \in B[0, M]$, i.e., the operator $A$ maps the ball $B[0, M]$ into itself.

Next, we prove that the operator $A$ is a compact one in the space $C[0,1]$
Providing the subscript $\varphi$ for $u$ we have

$$
\begin{aligned}
& u_{\varphi}(x)=\int_{0}^{1} G(x, t) \varphi(t) d t, \\
& u_{\varphi}^{\prime}(x)=\int_{0}^{1} G_{x}(x, t) \varphi(t) d t, \\
& u_{\varphi}^{\prime \prime}(x)=\int_{0}^{1} G_{x x}(x, t) \varphi(t) d t, \\
& u_{\varphi}^{\prime \prime \prime}(x)=\int_{0}^{1} G_{x x x}(x, t) \varphi(t) d t .
\end{aligned}
$$

According to [3] the integral operators in (26)-(29) which put function $\varphi \in C[0,1]$ in correspondence to the functions $u_{\varphi}, u_{\varphi}^{\prime}, u_{\varphi}^{\prime \prime}, u_{\varphi}^{\prime \prime \prime}$ are compact operators. Therefore, in view of the continuity of the function $f(x, u, y, v, z)$ the it is easy to deduce that the operator $A$ is compact operator in the space $C[0,1]$. Thus, $A$ is a compact operator from the closed ball $B[0, M]$ into itself. By the Schauder Fixed Point Theorem [4] the operator equation (19) has a solution. The estimates (23) indeed are the estimates (25). The theorem is proved.

Theorem 4. (Uniqueness of solution) Suppose that there exist numbers $M, c_{0}, c_{1}, c_{2}, c_{3} \geq 0$ such that

$$
\begin{aligned}
& \left|f\left(x, u_{2}, y_{2}, v_{2}, z_{2}\right)-f\left(x, u_{1}, y_{1}, v_{1}, z_{1}\right)\right| \leq \\
& c_{0}\left|u_{2}-u_{1}\right|+c_{1}\left|y_{2}-y_{1}\right|+c_{2}\left|v_{2}-v_{1}\right|+c_{3}\left|z_{2}-z_{1}\right| \\
& \text { for any }\left(x, u_{i}, y_{i}, v_{i}, z_{i}\right) \in D_{M}(i=1,2) \text { and } \\
& \qquad q=\frac{c_{0}}{24}+0,3577 c_{1}+\frac{2}{3} c_{2}+c_{3}<1 .
\end{aligned}
$$

Then the solution of the problem (11)-(12) is unique if it exists.

Proof. Now, let $\varphi_{1}, \varphi_{2} \in B[0, M]$ and $u_{1}, u_{2}$ be the solutions of the problem (11)-(12) for $\varphi_{1}, \varphi_{2}$ respectively.

We also denote 
$y_{i}=u_{i}^{\prime}, v_{i}=u^{\prime \prime}{ }_{i}, z_{i}=v_{i}^{\prime},(i=1,2)$. $)$.

Then, as induced above

$$
\left(x, u_{i}, y_{i}, v_{i}, z_{i}\right) \in D_{M}(i=1,2) \text { for } x \in[0,1] \text {. }
$$

Due to the estimates (25) we have

$\left\|u_{2}-u_{1}\right\| \leq \frac{1}{24}\left\|\varphi_{2}-\varphi_{1}\right\|,\left\|y_{2}-y_{1}\right\| \leq 0,3577\left\|\varphi_{2}-\varphi_{1}\right\|$,

$\left\|v_{2}-v_{1}\right\| \leq \frac{2}{3}\left\|\varphi_{2}-\varphi_{1}\right\|,\left\|z_{2}-z_{1}\right\| \leq\left\|\varphi_{2}-\varphi_{1}\right\|$.

Now from (20) and (30) it follows

$$
\begin{aligned}
& \left|A \varphi_{2}-A \varphi_{1}\right| \\
& =\left|f\left(x, u_{2}, y_{2}, v_{2}, z_{2}\right)-f\left(x, u_{1}, y_{1}, v_{1}, z_{1}\right)\right| \\
& \leq c_{0}\left|u_{2}-u_{1}\right|+c_{1}\left|y_{2}-y_{1}\right|+c_{2}\left|v_{2}-v_{1}\right|+c_{3}\left|z_{2}-z_{1}\right| .
\end{aligned}
$$

Using the estimate (32) we obtain

$$
\left\|A \varphi_{2}-A \varphi_{1}\right\| \leq \frac{c_{0}}{24}+0,3577 c_{1}+\frac{2}{3} c_{2}+c_{3}\left\|\varphi_{2}-\varphi_{1}\right\| .
$$

Therefore, $A$ is a contractive operator in $B[0, M]$ provided the condition (31) is satisfied. The theorem is proved.

The proposed approach can be used for some other nonlinear boundary value problems for ordinary and partial differential equations, such as the problem (7)-(8); (9)-(10).

\section{Conclusion}

In this paper, we study solvability of nonlinear boundary problems using the Green function. We have established the existence and uniqueness of a solution of the fully fourth order nonlinear boundary value problem. This method can be used for some other nonlinear boundary value problems for ordinary and partial differential equations. This is the direction of our research in the future.

\section{REFERENCES}

[1]. Y. A. Melnikov and M. Y. Melnikov, Green's Functions Construction and Applications, De Gruyter, 2012.

[2]. Q. A. Dang and T. K. Q. Ngo, "Existence results and iterative method for solving the cantilever beam equation with fully nonlinear term," Nonlinear Anal. Real World Appl., 36, pp. 56-68, 2017.

[3]. A. N. Kolmogorov and S. V. Fomin, Elements of the theory of functions and functional Analysis, Volume1: Metric and Normed Spaces, Graylockpress Rochester, 1957.

[4]. E. Zeidler, Nonlinear functional analysis and its applications, I: FixedPoint Theorems, Springer, 1986. 